\title{
Research on the nonlinear spherical percolation model with quadratic pressure gradient and its percolation characteristics
}

\author{
Ren-Shi Nie', Yong Ding ${ }^{2}$ \\ ${ }^{1}$ Petroleum Engineering Department, Southwest Petroleum University, Chengdu, China; nierenshi2000@163.com \\ ${ }^{2}$ Luliang Oilfield Production Company, Petrochina Xinjiang Oilfield Company, Kelamayi, China
}

Received 18 November 2009; revised 11 December 2009; accepted 30 December 2009.

\begin{abstract}
For bottom water reservoir and the reservoir with a thick oil formation, there exists partial penetration completion well and when the well products the oil flow in the porous media takes on spherical percolation. The nonlinear spherical flow equation with the quadratic gradient term is deduced in detail based on the mass conservation principle, and then it is found that the linear percolation is the approximation and simplification of nonlinear percolation. The nonlinear spherical percolation physical and mathematical model under different external boundaries is established, considering the effect of wellbore storage. By variable substitution, the flow equation is linearized, then the Laplace space analytic solution under different external boundaries is obtained and the real space solution is also gotten by use of the numerical inversion, so the pressure and the pressure derivative bi-logarithmic nonlinear spherical percolation type curves are drawn up at last. The characteristics of the nonlinear spherical percolation are analyzed, and it is found that the new nonlinear percolation type curves are evidently different from linear percolation type curves in shape and characteristics, the pressure curve and pressure derivative curve of nonlinear percolation deviate from those of linear percolation. The theoretical offset of the pressure and the pressure derivative between the linear and the nonlinear solution are analyzed, and it is also found that the influence of the quadratic pressure gradient is very distinct, especially for the low permeability and heavy oil reservoirs. The influence of the non-linear term upon the spreading of pressure is very distinct on the process of
\end{abstract}

percolation, and the nonlinear percolation law stands for the actual oil percolation law in reservoir, therefore the research on nonlinear percolation theory should be strengthened and reinforced.

Keywords: Nonlinear Spherical Percolation; Quadratic Pressure Gradient; Percolation Characteristics; Reservoir; Partial Penetration Completion Well; Mathematic Model

\section{INTRODUCTION}

So far, the research on nonlinear percolation has increasingly aroused widespread concern and attention. The nonlinear percolation is the modern development of a new direction [1]. Retaining the nonlinear term was proposed by Odeh A S [2]. He thought ignoring the quadratic gradient term would cause larger error in hydraulic fracturing, big pressure drop flow, DST and large pressure drop pulse testing. Bai M Q [3] considered that ignoring the quadratic gradient term in flow equation is equivalent to ignoring convection flow term in diffusion-convection equation. Wang Y [4] established the nonlinear flow model in poroelastic media. Chakrabarty $\mathrm{C}$ [5] derived the mathematical model with nonlinear diffusion equation and made the quantitative analysis of the quadratic term. Braeuning S [6] established the nonlinear radial flow model of the variable-rate well-test. Tong Dengke [7,8] solved the well test models of heterogeneous and dual porosity reservoir. Concerning the spherical flow, the linear spherical flow model was studied by William E. Brigham, Charles A. Kohlhaas and Mark A. Proett, et al. [9-11]. In their models, no nonlinear spherical flow model is found, so this paper presents the nonlinear spherical flow model and researches its percolation characteristics for partial penetration completion well in the formation. 


\section{DEDUCTION OF THE NONLINEAR SPHERICAL PERCOLATION EQUATION}

When single-phase fluid flow through porous medium, it would conform to the mass conservation principle, so by this principle the flow equation of continuity can be expressed by

$$
\frac{\partial}{\partial x}\left(\rho v_{x}\right)+\frac{\partial}{\partial y}\left(\rho v_{y}\right)+\frac{\partial}{\partial z}\left(\rho v_{z}\right)=-\frac{\partial}{\partial t}(\rho \phi)
$$

where: $v$ is flow velocity, $\mathrm{cm} / \mathrm{s} ; \rho$ is oil density, $\mathrm{g} / \mathrm{cm}^{3} ; t$ is flow time, $\mathrm{s} ; \varphi$ is rock porosity, fraction; $x, y, z$ represent the Cartesian coordinates.

If ignoring the impact of gravity and capillary forces, and the inertial resistance is not considered, it would conform to the Darcy's law, so the equation of motion is as follows

$$
v=-\frac{k}{\mu} \nabla p
$$

where: $k$ is rock permeability, $\mu \mathrm{m}^{2} ; \mu$ is fluid viscosity, $\mathrm{mPa} \cdot \mathrm{s} ; p$ is formation pressure, $\mathrm{MPa}$.

The fluid flow through porous medium is a process of percolation, and is also a state of constantly changing process, in which the parameters related to percolation are constantly changing with pressure and temperature. Usually the change of temperature in reservoir is inappreciable, so the flow is taken as isothermal flow. The rock and fluid are elastic and slightly compressible, the state equation of fluid and the state equation of rock are expressed as follows respectively

$$
\begin{aligned}
& \rho=\rho_{0} \mathrm{e}^{C_{\boldsymbol{\rho}}\left(p-p_{0}\right)} \\
& \phi=\phi_{0} \mathrm{e}^{C_{\mathrm{f}}\left(p-p_{0}\right)}
\end{aligned}
$$

where: $C_{\rho}$ is oil compressibility, $\mathrm{MPa}^{-1} ; C_{\mathrm{f}}$ is rock compressibility, $\mathrm{MPa}^{-1}$; the subscript " 0 " represents reference value, usually use the value in standard conditions.

Substitute Eq.(2) into Eq.(1)

$$
\begin{gathered}
\frac{\partial}{\partial x}\left(\rho \frac{k_{x}}{\mu} \frac{\partial p}{\partial x}\right)+\frac{\partial}{\partial y}\left(\rho \frac{k_{y}}{\mu} \frac{\partial p}{\partial y}\right)+ \\
\frac{\partial}{\partial z}\left(\rho \frac{k_{z}}{\mu} \frac{\partial p}{\partial z}\right)=\frac{\partial}{\partial t}(\rho \phi) \\
\frac{\partial}{\partial x}\left(\rho \frac{k_{x}}{\mu} \frac{\partial p}{\partial x}\right)=\frac{\rho k_{x}}{\mu} \frac{\partial^{2} p}{\partial x^{2}}+ \\
\frac{\rho}{\mu} \frac{\partial p}{\partial x} \frac{\partial k_{x}}{\partial x}+\frac{k_{x}}{\mu} \frac{\partial p}{\partial x} \frac{\partial \rho}{\partial x}
\end{gathered}
$$

Changing the form of Eq.(3)

$$
p=\frac{1}{C_{\rho}} \ln \rho-\frac{1}{C_{\rho}} \ln \rho_{0}+p_{0}
$$

$$
\begin{aligned}
& \frac{\partial p}{\partial x}=\frac{1}{\rho C_{\rho}} \frac{\partial \rho}{\partial x} \\
& \frac{\partial p}{\partial t}=\frac{1}{\rho C_{\rho}} \frac{\partial \rho}{\partial t}
\end{aligned}
$$

Substitute Eq.(8) into Eq.(6)

$$
\begin{gathered}
\frac{\partial}{\partial x}\left(\rho \frac{k_{x}}{\mu} \frac{\partial p}{\partial x}\right)=\frac{\rho k_{x}}{\mu} \frac{\partial^{2} p}{\partial x^{2}}+ \\
\frac{\rho}{\mu} \frac{\partial p}{\partial x} \frac{\partial k_{x}}{\partial x}+\frac{k_{x} \rho C_{\rho}}{\mu}\left(\frac{\partial p}{\partial x}\right)^{2}
\end{gathered}
$$

By the same method, the following two equations can be deduced

$$
\begin{gathered}
\frac{\partial}{\partial y}\left(\rho \frac{k_{y}}{\mu} \frac{\partial p}{\partial y}\right)=\frac{\rho k_{y}}{\mu} \frac{\partial^{2} p}{\partial y^{2}}+ \\
\frac{\rho}{\mu} \frac{\partial p}{\partial y} \frac{\partial k_{y}}{\partial y}+\frac{k_{y} \rho C_{\rho}}{\mu}\left(\frac{\partial p}{\partial y}\right)^{2} \\
\frac{\partial}{\partial z}\left(\rho \frac{k_{z}}{\mu} \frac{\partial p}{\partial z}\right)=\frac{\rho k_{z}}{\mu} \frac{\partial^{2} p}{\partial z^{2}}+ \\
\frac{\rho}{\mu} \frac{\partial p}{\partial z} \frac{\partial k_{z}}{\partial z}+\frac{k_{z} \rho C_{\rho}}{\mu}\left(\frac{\partial p}{\partial z}\right)^{2}
\end{gathered}
$$

Changing the form of Eq.(4)

$$
\begin{gathered}
p=\frac{1}{C_{\mathrm{f}}} \ln \phi-\frac{1}{C_{\mathrm{f}}} \ln \phi_{0}+p_{0} \\
\frac{\partial p}{\partial t}=\frac{1}{\phi C_{\mathrm{f}}} \frac{\partial \phi}{\partial t}
\end{gathered}
$$

Substitute Eq.(14) and Eq.(9) into Eq.(5), the right of Eq.(5) can be changed

$$
\begin{gathered}
\frac{\partial}{\partial t}(\rho \phi)=\phi \frac{\partial \rho}{\partial t}+\rho \frac{\partial \phi}{\partial t}=\rho \phi C_{\rho}+ \\
\rho \phi C_{\mathrm{f}} \frac{\partial p}{\partial t}=\rho \phi C_{\mathrm{t}} \frac{\partial p}{\partial t} \\
C_{\mathrm{t}}=C_{\rho}+C_{\mathrm{f}}
\end{gathered}
$$

where: $C_{\mathrm{t}}$ is total compressibility of rock and oil, $\mathrm{MPa}^{-1}$.

Substitute Eqs.(10)-(12) and Eq.(15) into Eq.(5), we have

$$
\begin{gathered}
\left(k_{x} \frac{\partial^{2} p}{\partial x^{2}}+k_{y} \frac{\partial^{2} p}{\partial y^{2}}+k_{z} \frac{\partial^{2} p}{\partial z^{2}}\right)+\left(\frac{\partial p}{\partial x} \frac{\partial k_{x}}{\partial x}+\right. \\
\left.\frac{\partial p}{\partial y} \frac{\partial k_{x}}{\partial y}+\frac{\partial p}{\partial z} \frac{\partial k_{x}}{\partial z}\right)+C_{\rho}\left[k_{x}\left(\frac{\partial p}{\partial x}\right)^{2}+\right.
\end{gathered}
$$




$$
\left.k_{y}\left(\frac{\partial p}{\partial y}\right)^{2}+k_{z}\left(\frac{\partial p}{\partial z}\right)^{2}\right]=\mu \phi C_{\mathrm{t}} \frac{\partial p}{\partial t}
$$

If the permeability is isotropic and constant, $\partial k_{x} / \partial r=0, \partial k_{y} / \partial r=0, \partial k_{y} / \partial r=0$, the Eq.(17) becomes

$$
\begin{gathered}
\left(\frac{\partial^{2} p}{\partial x^{2}}+\frac{\partial^{2} p}{\partial y^{2}}+\frac{\partial^{2} p}{\partial z^{2}}\right)+C_{\rho}\left[\left(\frac{\partial p}{\partial x}\right)^{2}+\right. \\
\left.\left(\frac{\partial p}{\partial y}\right)^{2}+\left(\frac{\partial p}{\partial z}\right)^{2}\right]=\frac{\mu \phi C_{\mathrm{t}}}{k} \frac{\partial p}{\partial t}
\end{gathered}
$$

Eq.(18) is the governing differential equation in Cartesian coordinates, the equation in radial spherical coordinates becomes

$$
\frac{1}{r^{2}} \frac{\partial}{\partial r}\left(r^{2} \frac{\partial p}{\partial r}\right)+C_{\rho}\left(\frac{\partial p}{\partial r}\right)^{2}=\frac{\mu \phi C_{\mathrm{t}}}{k} \frac{\partial p}{\partial t}
$$

where, $r$ represents the radial spherical coordinates.

Eq.(19) is the nonlinear flow governing partial differential equation with quadratic pressure gradient term. We call the second power of the pressure gradient as quadratic pressure gradient.

The function $\exp (x)$ by use of Maclaurin series expansion is written by

$$
\exp (x)=1+x+x^{2} / 2+\cdots+x^{n} / n !+\cdots
$$

If we use Maclaurin series expansion for Eqs.(3) and (4) and neglect the second order and the above higher order item, the Eqs.(3) and (4) can be rewritten by Eqs. (21) and (22) respectively

$$
\begin{gathered}
\rho=\rho_{0}\left[1+C_{\rho}\left(p-p_{0}\right)\right] \\
\phi=\phi_{0}\left[1+C_{\mathrm{f}}\left(p-p_{0}\right)\right]
\end{gathered}
$$

The appearance of quadratic pressure gradient term is simply because that we didn't make any simplification for the state Eqs. (3) and (4) in the deduction of the flow governing partial differential equation. If we use Eqs. (21) and (22), instead of Eqs.(3) and (4), in the deduction of the flow governing partial differential equation, the quadratic pressure gradient term will not come up, and the deduced flow equation is the conventional linear flow equation, which is shown in almost any percolation mechanics books and papers, so the deduction of the linear flow equation is certainly omitted here. Owing to the existence of quadratic pressure gradient, the flow equation takes on nonlinear properties. Therefore it can be safely concluded that the conventional linear flow equation is the approximation and simplification of nonlinear flow equation with quadratic pressure gradient term, and that the nonlinear percolation law stands for the actual flow law of oil in reservoir.

\section{3 .SPHERICAL PERCOLATION MODELS AND ITS SOLUTION}

\subsection{Physical Model}

For bottom water reservoir, the position of drilling and completion of oil well is usually in the top of the oil formation, the flow diagram shown in Figure 1. For some reservoirs, the oil formation is very thick, the position of drilling and completion of well is usually in the middle of the formation, the flow diagram shown in Figure 2. For the two actual situations, the oil flow in the porous media is in the form of spherical percolation.

Physical model assumptions are as follows:

1) A single well with partial penetration completion in the formation like Figure 1 or Figure 2 products at constant rate, the external boundary may be infinite or closed or constant pressure;

2) The rock and the single-phase fluid are slightly compressible, a constant compressibility;

3) Isothermal and Darcy flow, the permeability and porosity of isotropic properties;

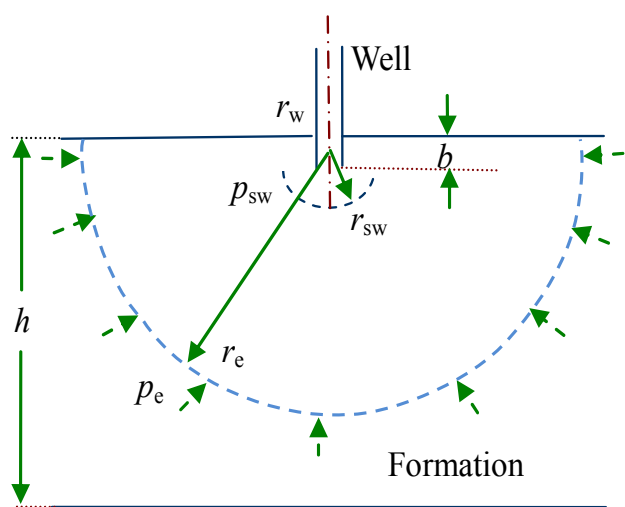

Figure 1. Spherical flow diagram for well completion position in the top of the formation.

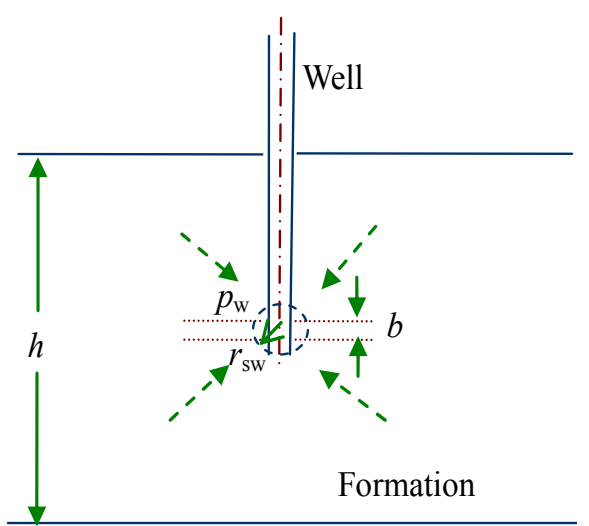

Figure 2. Spherical flow diagram for well completion position in the middle of the formation. 
4) Considering wellbore storage effects (in the beginning of opening well, the fluid stored in the wellbore starts to flow, the oil in the formation does not flow);

5 ) At time $t=0$, pressure is uniformly distributed in the reservoir, equal to the initial pressure $p_{i}$;

6) Ignoring the impact of gravity and capillary forces.

\subsection{Mathematic Model}

The governing differential equation in radial spherical coordinate system

$$
\frac{1}{r^{2}} \frac{\partial}{\partial r}\left(r^{2} \frac{\partial p}{\partial r}\right)+C_{\rho}\left(\frac{\partial p}{\partial r}\right)^{2}=\frac{\mu \phi C_{\mathrm{t}}}{3.6 k} \frac{\partial p}{\partial t}
$$

where: $r$ is the radial spherical distance from well, $\mathrm{m}$; the unit of well production time $(t)$ becomes " $h$ ", so the coefficient "3.6" appears in the Eq.(23).

Initial conditions

$$
\left.p\right|_{t=0}=p_{\mathrm{i}}
$$

where, $p_{\mathrm{i}}$ is initial formation pressure, $\mathrm{MPa}$.

Inner boundary condition

$$
\begin{gathered}
\left.\frac{k}{\mu}\left(r^{2} \frac{\partial p}{\partial r}\right)\right|_{r=r_{\mathrm{sw}}}=0.921 \times 10^{-3} q B+ \\
0.022105 C_{\mathrm{s}} \frac{\mathrm{d} p_{w}}{\mathrm{~d} t}
\end{gathered}
$$

where: the $r_{\mathrm{ws}}$ is called the pseudo well radius of radial spherical flow, and $r_{\mathrm{ws}}=b /\left(2 \ln \left(b / r_{\mathrm{w}}\right)\right)$ [12], $b$ is the formation penetration thickness of well completion, $\mathrm{m} ; r_{\mathrm{w}}$ is the real well radius $\mathrm{m} ; q$ is oil rate at wellhead, $\mathrm{m}^{3} / \mathrm{d} ; B$ is oil volume factor, dimensionless; $C_{\mathrm{s}}$ is wellbore storage coefficient, $\mathrm{m}^{3} / \mathrm{MPa} ; p_{\mathrm{w}}$ is wellbore pressure, $\mathrm{MPa}$.

External boundary condition

$$
\begin{gathered}
\lim _{r \rightarrow \infty} p=p_{\mathrm{i}} \text { (infinite) } \\
\left.p\right|_{r=r_{\mathrm{e}}}=p_{\mathrm{i}}(\text { constant pressure) } \\
\left.\frac{\partial p}{\partial r}\right|_{r=r_{\mathrm{e}}}=0 \text { (closed) }
\end{gathered}
$$

where, $r_{\mathrm{e}}$ is external boundary radius, $\mathrm{m}$.

\subsection{Solution to Mathematic Model}

The dimensionless definitions are as follows:

Dimensionless pressure

$$
p_{\mathrm{D}}=k r_{\mathrm{sw}}\left(p_{\mathrm{i}}-p\right) /\left(0.921 \times 10^{-3} \mu q B\right) ;
$$

Dimensionless radius based on pseudo spherical flow radius $r_{\mathrm{D}}=r / r_{\mathrm{sw}}$;

Dimensionless wellbore storage coefficient

$$
C_{\mathrm{D}}=C_{\mathrm{s}} /\left(3.1416 \phi C_{\mathrm{t}} r_{\mathrm{sw}}^{3}\right)
$$

Dimensionless time $t_{\mathrm{D}}=3.6 \mathrm{kt} /\left(\phi \mu C_{\mathrm{t}} r_{\mathrm{sw}}{ }^{2}\right)$;

Dimensionless quadratic pressure gradient coefficient

$$
\beta=0.921 \times 10^{-3} q B \mu C_{\rho} /\left(k r_{\mathrm{sw}}\right)
$$

The dimensionless model is as follows:

The governing differential equation in radial spherical coordinate system

$$
\frac{\partial^{2} p_{\mathrm{D}}}{\partial r_{\mathrm{D}}{ }^{2}}+\frac{2}{r_{\mathrm{D}}} \frac{\partial p_{\mathrm{D}}}{\partial r_{\mathrm{D}}}-\beta\left(\frac{\partial p_{\mathrm{D}}}{\partial r_{\mathrm{D}}}\right)^{2}=\frac{\partial p_{\mathrm{D}}}{\partial t_{\mathrm{D}}}
$$

Initial conditions

$$
\left.p_{\mathrm{D}}\right|_{t_{\mathrm{D}}=0}=0
$$

Inner boundary condition

$$
C_{\mathrm{D}} \frac{\mathrm{d} p_{\mathrm{wD}}}{\mathrm{d} t_{\mathrm{D}}}-\left.\left(r_{\mathrm{D}} \frac{\partial p_{\mathrm{D}}}{\partial r_{\mathrm{D}}}\right)\right|_{r_{\mathrm{D}}=1}=1
$$

External boundary condition

$$
\begin{gathered}
\lim _{r_{\mathrm{D}} \rightarrow \infty} p_{\mathrm{D}}\left(r_{\mathrm{D}}, t_{\mathrm{D}}\right)=0 \text { (infinite) } \\
\left.p_{\mathrm{D}}\right|_{r_{\mathrm{D}}=r_{\mathrm{eD}}}=0 \text { (constant pressure) } \\
\left.\frac{\partial p_{\mathrm{D}}}{\partial r_{\mathrm{D}}}\right|_{r=r_{\mathrm{eD}}}=0 \text { (closed) }
\end{gathered}
$$

Take

$$
p_{\mathrm{D}}=-\frac{1}{\beta} \ln x
$$

where, $x$ is substitution variable between variables.

Making the upper variable substitutions for Eqs. (29-34), the model can be converted to

The governing differential equation

$$
\left(\frac{\partial^{2} x}{\partial r_{\mathrm{D}}^{2}}+\frac{2}{r_{\mathrm{D}}} \frac{\partial x}{\partial r_{\mathrm{D}}}\right)=\frac{\partial x}{\partial t_{\mathrm{D}}}
$$

Initial conditions

$$
\left.x\right|_{t_{\mathrm{D}}=0}=1
$$

Inner boundary condition

$$
\left.\left(C_{\mathrm{D}} \frac{\partial x}{\partial t_{\mathrm{D}}}-\frac{\partial x}{\partial r_{\mathrm{D}}}+\beta x\right)\right|_{r_{\mathrm{D}}=1}=0
$$

External boundary condition

$$
\begin{gathered}
\lim _{r_{\mathrm{D}} \rightarrow \infty} x\left(r_{\mathrm{D}}, t_{\mathrm{D}}\right)=1 \text { (infinite) } \\
\left.x\right|_{r_{\mathrm{D}}=r_{\mathrm{eD}}}=1 \text { (constant pressure) } \\
\left.\frac{\partial x}{\partial r_{\mathrm{D}}}\right|_{r_{\mathrm{D}}=r_{\mathrm{eD}}}=0 \text { (closed) }
\end{gathered}
$$

Take 


$$
x=y / r_{\mathrm{D}}+1
$$

where, $y$ is substitution variable between variables.

Making the upper variable substitutions for Eqs. (36-41), the model can be converted to

The governing differential equation

$$
\frac{\partial^{2} y}{\partial r_{\mathrm{D}}^{2}}=\frac{\partial y}{\partial t_{\mathrm{D}}}
$$

Initial conditions

$$
\left.y\right|_{t_{\mathrm{D}}=0}=0
$$

Inner boundary condition

$$
\left.\left[C_{\mathrm{D}} \frac{\partial y}{\partial t_{\mathrm{D}}}-\frac{\partial y}{\partial r_{\mathrm{D}}}+(\beta+1) y\right]\right|_{\mathrm{r}_{\mathrm{D}}=1}=-\beta
$$

External boundary condition

$$
\begin{gathered}
\lim _{r_{\mathrm{D}} \rightarrow \infty} y=0 \text { (infinite) } \\
\left.y\right|_{r_{\mathrm{D}}=r_{\mathrm{eD}}}=0 \text { (constant pressure) } \\
\left.\left(\frac{\partial y}{\partial r_{\mathrm{D}}}-y \frac{1}{r_{\mathrm{D}}}\right)\right|_{r_{\mathrm{D}}=r_{\mathrm{eD}}}=0 \text { (closed) }
\end{gathered}
$$

Introduce the Laplace transform based on $t_{\mathrm{D}}$, that is

$$
L\left[p_{\mathrm{D}}\left(t_{\mathrm{D}}\right)\right]=\bar{p}_{\mathrm{D}}(z)=\int_{0}^{\infty} p_{\mathrm{D}}\left(t_{\mathrm{D}}\right) \mathrm{e}^{-z t_{\mathrm{D}}} \mathrm{d} t_{\mathrm{D}}
$$

where, $z$ is Laplace space variable.

So, making the Laplace transform of Eqs.(43-48), the model becomes:

The governing differential equation in Laplace space

$$
\frac{\mathrm{d}^{2} \bar{y}}{\mathrm{~d} r_{\mathrm{D}}^{2}}-z \bar{y}=0
$$

Inner boundary condition in Laplace space

$$
\left[\left(C_{\mathrm{D}} z+\beta+1\right) \bar{y}-\frac{\mathrm{d} \bar{y}}{\mathrm{~d} r_{\mathrm{D}}}\right]_{r_{\mathrm{D}}=1}=-\frac{\beta}{z}
$$

External boundary condition in Laplace space

$$
\begin{gathered}
\lim _{r_{\mathrm{D}} \rightarrow \infty} \bar{y}=0 \text { (infinite) } \\
\left.\bar{y}\right|_{r_{\mathrm{D}}=r_{\mathrm{CD}}}=0 \text { (constant pressure) } \\
\left.\left(\frac{\partial \bar{y}}{\partial r_{\mathrm{D}}}-\bar{y} \frac{1}{r_{\mathrm{D}}}\right)\right|_{r_{\mathrm{D}}=r_{\mathrm{CD}}}=0 \text { (closed) }
\end{gathered}
$$

The general solution of Eq.(50) can be expressed by

$$
\bar{y}=A \mathrm{e}^{\sqrt{z} r_{\mathrm{D}}}+B \mathrm{e}^{-\sqrt{z} r_{\mathrm{D}}}
$$

For infinite boundary:

Substitute Eq.(55) into Eq.(52), have

$$
A=0
$$

So the general solution of Eq.(50) becomes

$$
\bar{y}=B \mathrm{e}^{-\sqrt{z} r_{\mathrm{D}}}
$$

Substitute Eq.(57) into Eq.(51), have

$$
B=-\frac{\beta}{z\left(C_{\mathrm{D}} z+\beta+1+\sqrt{z}\right) \mathrm{e}^{-\sqrt{z}}}
$$

The general solution of Eq.(50) can be got by

$$
\bar{y}=-\frac{\beta}{z\left(C_{\mathrm{D}} z+\beta+1+\sqrt{z}\right) \mathrm{e}^{-\sqrt{z}}} \mathrm{e}^{-\sqrt{z} r_{\mathrm{D}}}
$$

At the wellbore bottom, $r=r_{\mathrm{w}}, r_{\mathrm{D}}=1, p=p_{\mathrm{w}}, p_{\mathrm{D}}=p_{\mathrm{wD}}$, $x=x_{\mathrm{w}}, y=y_{\mathrm{w}}$, therefore, the solution of the spherical percolation model with infinite external boundary in Laplace space can be got by

$$
\bar{y}_{\mathrm{w}}=\left.\bar{y}\right|_{r_{\mathrm{D}}=1}=-\frac{\beta}{z\left(C_{\mathrm{D}} z+\beta+1+\sqrt{z}\right)}
$$

The real space solution $y_{\mathrm{w}}$ and the derivative $\left(\mathrm{d} y_{\mathrm{w}} / \mathrm{d} t_{\mathrm{D}}\right)$ can be easily obtained by use of Stehfest numerical inversion [13] for Eq.(60). Substitute the values of inversion into variable substitution relationships, Eq.(35) and Eq.(42), so the real space solution $p_{\mathrm{wD}}$ and the derivative $\left(\mathrm{d} p_{\mathrm{wD}} / \mathrm{d} t_{\mathrm{D}}\right)$ can be certainly gained. Accordingly, the pressure and the pressure derivative bi-logarithmic type curves of nonlinear spherical percolation can be drawn up (see Figure 3).

For constant pressure boundary:

At the wellbore bottom $r_{\mathrm{D}}=1, y=y_{\mathrm{w}}$, the Eq.(55) becomes

$$
\mathrm{e}^{\sqrt{z}} \cdot A+\mathrm{e}^{-\sqrt{z}} \cdot B-\bar{y}_{\mathrm{w}}=0
$$

Substitute Eq.(61) into Eq.(51) and Eq.(53), have respectively

$$
\begin{array}{r}
-\sqrt{z} \mathrm{e}^{\sqrt{z}} \cdot A+\sqrt{z} \mathrm{e}^{-\sqrt{z}} \cdot B+ \\
\left(C_{\mathrm{D}} z+\beta+1\right) \bar{y}_{\mathrm{w}}=-\frac{\beta}{z}
\end{array}
$$

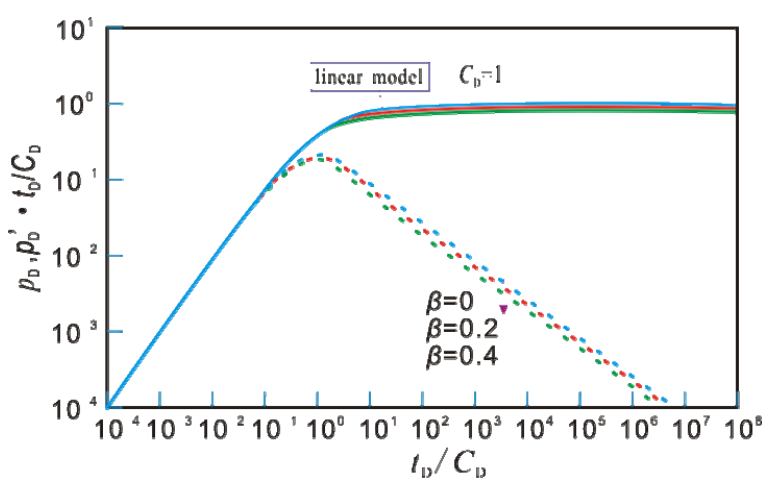

Figure 3. Type curves of nonlinear spherical percolation affected by $\beta$ under infinite external boundary. 


$$
\mathrm{e}^{\sqrt{z} r_{\mathrm{eD}}} \cdot A+\mathrm{e}^{-\sqrt{z} r_{\mathrm{eD}}} \cdot B=0
$$

For closed boundary:

Substitute Eq.(61) into Eq.(54), have

$$
\left(\sqrt{z} r_{\mathrm{eD}}-1\right) \mathrm{e}^{\sqrt{z} r_{\mathrm{eD}}} A-\left(\sqrt{z} r_{\mathrm{eD}}+1\right) \mathrm{e}^{-\sqrt{z} r_{\mathrm{eD}}} B=0
$$

Combining Eqs.(61-64), the coefficients $A$ and $B$ and the function at wellbore $\bar{y}_{\mathrm{w}}$ in Laplace space can be easily obtained by use of some linear algebra method (such as Gauss-Jordan reduction, etc), then nonlinear spherical percolation type curves can also be drawn up (see Figure 4 and Figure 5) by use of the same method.

\section{CHARACTERISTICS OF THE NONLINEAR PERCOLATION}

\subsection{Parameter Sensitivity Analysis to Type Curves}

Figure 3 shows the type curves of nonlinear spherical percolation affected by $\beta$ under infinite external boundary. Can be seen from the figure, the curves vary with the value of the dimensionless quadratic pressure gradient coefficient $\beta$ (from up to down, $\beta=0,0.2,0.4$ ), when $\beta=0$ it is just the curve of linear percolation model. It can be easily seen that the curves have the trait of unit slope in the wellbore storage stage, which shows that there is no influence of quadratic pressure gradient in this flow stage, and that the location of the pressure and the pressure derivative curves is lower than that of the conventional linear model curve in the stage of infinite-acting radial spherical flow. The bigger the $\beta$ is, the greater the offset is.

Figure 4 and Figure 5 show the type curves of nonlinear spherical percolation affected by $\beta$ under constant pressure external boundary and closed external boundary respectively. Can be seen from the figures, the trait of unit slope in the wellbore storage stage still exist and there still exists a offset due to the effect of $\beta$, but in the late flow stage of boundary response the pressure derivative curves is going down until focusing on a point for constant pressure boundary and the pressure derivative curves is going up until focusing on a line together with the pressure curves for closed boundary, which is completely different from Figure 1.

Figure 6 shows the type curves of nonlinear spherical percolation affected by $C_{\mathrm{D}}$ under infinite external boundary. Can be seen from the figure, the curves vary with the value of the dimensionless wellbore storage coefficient $C_{\mathrm{D}}$, and the bigger the $C_{\mathrm{D}}$ is, the lower thepressure derivative curve is. Figure 7 shows the type curves of nonlinear spherical percolation affected by $r_{\mathrm{eD}}$ under different external boundaries. Can be seen from the figure, the curves vary with the value of the dimen-

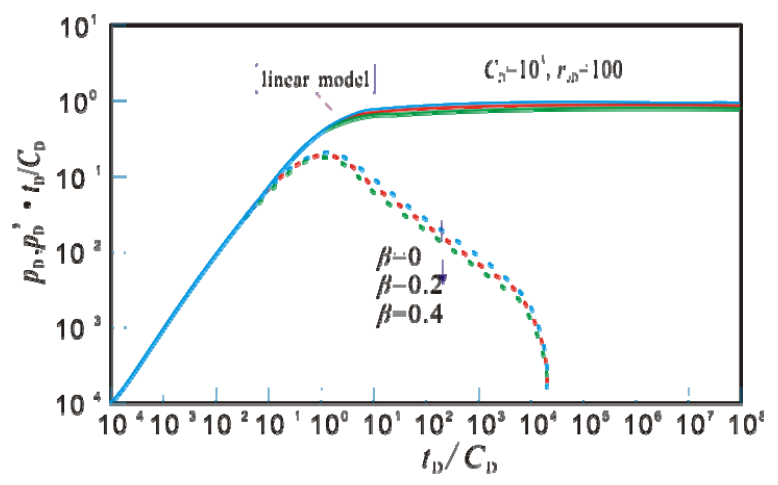

Figure 4. Type curves of nonlinear spherical percolation affected by $\beta$ under constant pressure external boundary.

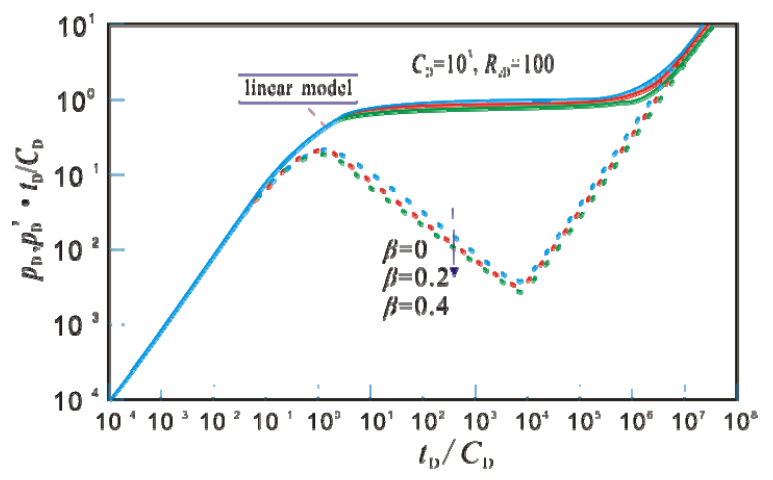

Figure 5. Type curves of nonlinear spherical percolation affected by $\beta$ under closed external boundary.

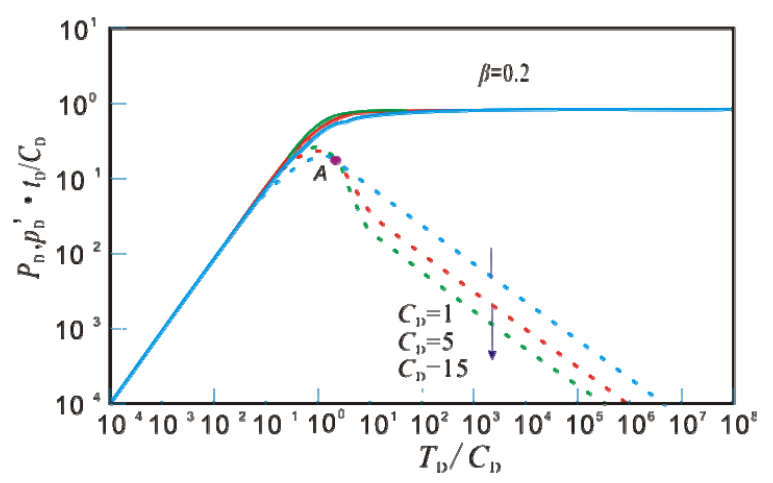

Figure 6. Type curves of nonlinear spherical percolation affected by $C_{\mathrm{D}}$.

sionless radial spherical radius $r_{\mathrm{eD}}$, and the bigger the $r_{\mathrm{eD}}$ is, the later the time of going up or going down is.

According to the definition of $\beta$ and the probable values of $\beta$ (Table 1), it is clearly demonstrated that $\beta$ is proportional to oil viscosity $\mu$, and inversely proportional to formation permeability $k$. So there is usually a bigger $\beta$ for the low permeability, heavy oil reservoirs, and the influence of the quadratic pressure gradient nonlinear term is very distinct, the quadratic pressure gradient should not be neglected. For the fixed group of parameters $\left(q, B, C_{\rho}\right)$, the speed of pressure wave propagation 


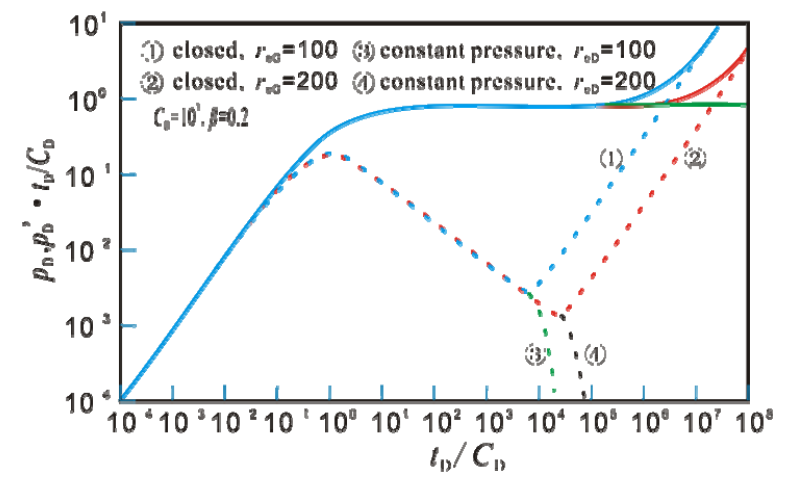

Figure 7. Type curves of nonlinear spherical percolation affected by $r_{\mathrm{eD}}$.

Table 1. The probable values of $\beta$.

\begin{tabular}{ccc}
\hline$k /\left(\times 10^{-3} \mu \mathrm{m}^{2}\right)$ & $\mu /(\mathrm{mPa} \cdot \mathrm{s})$ & $\beta$ \\
\hline 100 & 25 & 0.0058 \\
10 & 25 & 0.0580 \\
1 & 25 & 0.5800 \\
100 & 100 & 0.0230 \\
10 & 100 & 0.2300 \\
1 & 100 & 2.3000 \\
\hline
\end{tabular}

becomes slower when $k / \mu$ decreases with the increasing of $\beta$. Compared with the conventional linear model, given a fixed production time, pressure decline slows down and the speed of decline is inversely proportional to $\beta$, which is completely accordant with the theoretical curves as Figures 3-5. In conclusion, for a concrete reservoir, due to the effect of quadratic pressure gradient, compared with conventional linear model, the time of stable production of the nonlinear model is prolonged on condition that the same decline of reservoir pressure.

\subsection{The Influence Analysis of Nonlinear Term}

Table 2 and Table 3 exhibit the results of the pressure offset and pressure derivative offset analyses vs. Figure 3. As shown the data in these tables, the offset increase with the increasing of time at a constant $\beta$, and pressure derivative relative offset is greater than the pressure relative offset at a fixed time. From the data in the table, it is found that the impact of the quadratic gradient is extremely intense when time is particularly long and the quadratic coefficient $\beta$ is particularly large, so the quadratic pressure gradient should be retained in flow equation. After all, the nonlinear percolation law is the actual flow law of oil in porous medium, so the research on the nonlinear percolation model and its percolation law with quadratic pressure gradient should be strengthened and reinforced.

\section{CONCLUSIONS}

In this paper it is demonstrated that the quadratic pressure gradient has some distinct influence on the wellbore1) The linear percolation is the approximation and simplification of nonlinear percolation with quadratic pressure gradient term.

Table 2. The offset analysis of nonlinear $\operatorname{term}(\beta=0.2)$.

\begin{tabular}{|c|c|c|c|c|c|c|c|c|}
\hline \multirow{2}{*}{$t_{\mathrm{D}} / C_{\mathrm{D}}$} & \multicolumn{2}{|c|}{$P_{\mathrm{wD}}$} & \multirow{2}{*}{ offset } & \multirow{2}{*}{$\begin{array}{l}\text { relative } \\
\text { offset } / \%\end{array}$} & \multicolumn{2}{|c|}{$P^{\prime}{ }_{\mathrm{wD}} \cdot t_{\mathrm{D}} / C_{\mathrm{D}}$} & \multirow{2}{*}{ offset } & \multirow{2}{*}{$\begin{array}{c}\text { relative } \\
\text { offset } / \%\end{array}$} \\
\hline & linear & nonlinear & & & linear & nonlinear & & \\
\hline $10^{2}$ & 0.943292 & 0.864578 & 0.078714 & 8.34 & 0.029071 & 0.024059 & 0.005012 & 17.24 \\
\hline $10^{3}$ & 0.982148 & 0.896753 & 0.085395 & 8.69 & 0.008939 & 0.007390 & 0.001549 & 17.33 \\
\hline $10^{4}$ & 0.994357 & 0.906908 & 0.087449 & 8.79 & 0.002804 & 0.002312 & 0.000492 & 17.55 \\
\hline $10^{5}$ & 0.998216 & 0.910121 & 0.088095 & 8.83 & 0.000886 & 0.000728 & 0.000158 & 17.83 \\
\hline
\end{tabular}

Table 3. The offset analysis of nonlinear $\operatorname{term}(\beta=0.4)$.

\begin{tabular}{|c|c|c|c|c|c|c|c|c|}
\hline \multirow{2}{*}{$t_{\mathrm{D}} / C_{\mathrm{D}}$} & \multicolumn{2}{|c|}{$P_{\mathrm{wD}}$} & \multirow{2}{*}{ offset } & \multirow{2}{*}{$\begin{array}{l}\text { relative } \\
\text { offset } / \%\end{array}$} & \multicolumn{2}{|c|}{$P^{\prime}{ }_{\mathrm{wD}} \cdot t_{\mathrm{D}} / C_{\mathrm{D}}$} & \multirow{2}{*}{ offset } & \multirow{2}{*}{$\begin{array}{c}\text { relative } \\
\text { offset } / \%\end{array}$} \\
\hline & linear & nonlinear & & & linear & nonlinear & & \\
\hline $10^{2}$ & 0.943292 & 0.801013 & 0.142279 & 15.08 & 0.029071 & 0.020499 & 0.008572 & 29.49 \\
\hline $10^{3}$ & 0.982148 & 0.828462 & 0.153686 & 15.65 & 0.008939 & 0.006302 & 0.002637 & 29.50 \\
\hline $10^{4}$ & 0.994357 & 0.837153 & 0.157204 & 15.81 & 0.002804 & 0.001970 & 0.000834 & 29.74 \\
\hline $10^{5}$ & 0.998216 & 0.839906 & 0.158310 & 15.86 & 0.000886 & 0.000622 & 0.000264 & 29.80 \\
\hline
\end{tabular}


2) The new-style type curves of nonlinear spherical percolation with quadratic pressure gradient effect in shape and characteristics are obviously different from the type curves of linear model, the location of the pressure and the pressure derivative curves is lower than that of the conventional linear model curve.

3) The type curves are affected by the quadratic gradient coefficient $\beta$, the offset of pressure and pressure derivative is directly proportional to $\beta$ and time.

4) For a concrete reservoir, due to the effect of quadratic pressure gradient, compared with conventional linear model, the time of stable production of the nonlinear model is prolonged on condition that the same decline of reservoir pressure.

5) The impact of the quadratic pressure gradient under certain conditions is extremely intense, especially for the low permeability and heavy oil reservoirs, and the quadratic pressure gradient term should not be neglected and should be retained in flow equation.

6) The nonlinear percolation law is the actual flow law of oil in porous medium, so the research on the nonlinear flow model and its application with quadratic pressure gradient should be strengthened and reinforced.

\section{REFERENCES}

[1] Yan, B.S. and Ge, J.L. (2003) New advances of modern reservoir and fluid flow in porous media. Journal of Southwest Petroleum Institute, 25(1), 29-32, (in Chinese).

[2] Odeh, A.S and Babu, D.K. (1998) Comprising of solutions for the nonlinear and linearized diffusion equations. SPE Reservoir Engineering, 3(4), 1202-1206.
[3] Bai, M.Q. and Roegiers, J.C. (1994) A nonlinear dual porosity model. Appl Math Moclellin, 18(9), 602-610.

[4] Wang, Y. and Dusseault, M.B. (1991) The effect of quadratic gradient terms on the borehole solution in poroelastic media. Water Resource Research, 27(12), 3215-3223.

[5] Chakrabarty, C., Farouq, A.S.M. and Tortike, W.S. (1993) Analytical solutions for radial pressure distribution including the effects of the quadratic gradient term. Water Resource Research, 29(4), 1171-1177.

[6] Braeuning, S., Jelmert, T.A. and Sven, A.V. (1998) The effect of the quadratic gradient term on variable-rate well-tests, Journal of Petroleum Science and Engineering, 21(2), 203-222.

[7] Tong, D.K. (2003) The fluid mechanics of nonlinear flow in porous media. Beijing: Petroleum Industry Press in Chinese, (in Chinese).

[8] Tong, D.K., Zhang, Q.H. and Wang, R.H. (2005) Exact solution and its behavior characteristic of the nonlinear dual-porosity model. Applied Mathematic and Mechanics, 26(10), 1161-1167, (in Chinese).

[9] William, E.B., James, M. and Peden, K.F.N. (1980) The analysis of spherical flow with wellbore storage. SPE 9294.

[10] Charles, A.K. and William, A.A. (1982) Application of linear spherical flow analysis techniques to field problems-case studies. SPE 11088.

[11] Mark, A. and Proett, W.C.C. (1998) New exact spherical flow solution with storage and skin for early-time interpretation with applications to wireline formation and early-evaluation drillstem testing. SPE 49140.

[12] Joseph, J.A. and Koederitz, L.F. (1985) Unsready-state spherical flow with storage and skin. SPEJ, 25(6), 804-822.

[13] Stehfest H. (1970) Numerical inversion of Laplace transform algorithm 368, Communication of the ACM, 13(1), 47-49. 